\title{
Investigating the potential role of non-v/s genes on linear plasmid 28-1 in virulence and persistence by Borrelia burgdorferi
}

\author{
Petronella R. Hove Magunda ${ }^{1,2}$ and Troy Bankhead ${ }^{1,2^{*}}$ (D)
}

\begin{abstract}
Background: The Ip28-1 plasmid is required for persistent infection by the Lyme disease spirochete, Borrelia burgdorferi. Mutational studies on this plasmid have shown that the v/s locus is important for antigenic variation of the VIsE lipoprotein that leads to immune evasion and persistence. However, it is still unknown whether the v/s system is the only genetic locus on this plasmid necessary for long-term infection, and thus the potential role of non-v/s genes on Ip28-1 in virulence and persistence is yet to be fully determined. Despite extensive mutational analyses, two Ip28-1 regions containing the ORFs bbf19 - bbf22 and bbf27 - bbf30 have not yet been mutated in their entirety.

Results: In this study, we set out to establish if these unstudied regions of Ip28-1 play a role in spirochete persistence. Results show that the generated mutants were fully infectious in immunocompetent mice, and were able to persist for 91 days following infection. Following this finding, ospC expression by these mutants was determined, as it has been reported that spirochetes lacking Ip28-1 fail to downregulate expression of this lipoprotein leading to immune clearance. Data presented here failed to show a definitive difference in ospC expression levels during host infection when the mutants were compared to the wild type.

Conclusions: Overall, the results strongly suggest that non-v/s genes residing on Ip28-1 do not play a role in spirochete persistence during infection of the mammalian host, and that the regions under study are likely not involved in the regulation of $0 s p C$ expression. In conjunction with previous studies involving mutation of non-v/s loci on Ip28-1, these findings suggest that the v/s locus is likely the sole genetic element on this plasmid responsible for immune evasion and persistence exhibited by the Lyme disease pathogen.
\end{abstract}

Keywords: Borrelia, Lyme disease, Linear plasmid 28-1, Persistence, Immune evasion

\section{Background}

Lyme disease is caused by infection with the ticktransmitted spirochete, Borrelia burgdorferi. It is currently the most common vector-borne disease in the northern hemisphere, occurring in parts of North America, Europe and Asia [1]. Persistent host infection by B. burgdorferi has been linked to the presence of the 28 kilobase linear plasmid, lp28-1, which harbors 32 open reading frames that are designated $b b f 01$ to $b b f 32$ ([2, 3]; Fig. 1). Clones lacking lp28-1 exhibit an intermediate infectivity

\footnotetext{
* Correspondence: tbankhead@vetmed.wsu.edu

'Department of Veterinary Microbiology and Pathology, Washington State University, Pullman, WA, USA

${ }^{2}$ Paul G. Allen School for Global Animal Health, Washington State University, Pullman, WA, USA
}

(c) 2016 The Author(s). Open Access This article is distributed under the terms of the Creative Commons Attribution 4.0 International License (http://creativecommons.org/licenses/by/4.0/), which permits unrestricted use, distribution, and reproduction in any medium, provided you give appropriate credit to the original author(s) and the source, provide a link to the Creative Commons license, and indicate if changes were made. The Creative Commons Public Domain Dedication waiver (http://creativecommons.org/publicdomain/zero/1.0/) applies to the data made available in this article, unless otherwise stated.

phenotype, whereby these spirochetes are able to disseminate to tissue sites but are unable to persist in the murine host [4-6]. Notably, these same clones are capable of long-term survival in severe-combined immunodeficient (SCID) mice that lack an effective antibody response $[4,7]$. Studies involving telomere-mediated deletion of a $10 \mathrm{~kb}$ region (bbf31-bbf32) containing the VMP-like sequence $(\mathrm{vls})$ locus responsible for antigenic variation of a $v l s$-expressed (VlsE) lipoprotein found that these mutant spirochetes are cleared in immunocompetent mice by day 21 post infection, matching the phenotype observed with the complete loss of lp28-1 [8,9].

Although numerous studies have been conducted on the importance of the $v l s$ locus, it has not been fully determined whether other genes on 1p28-1 play a role in 


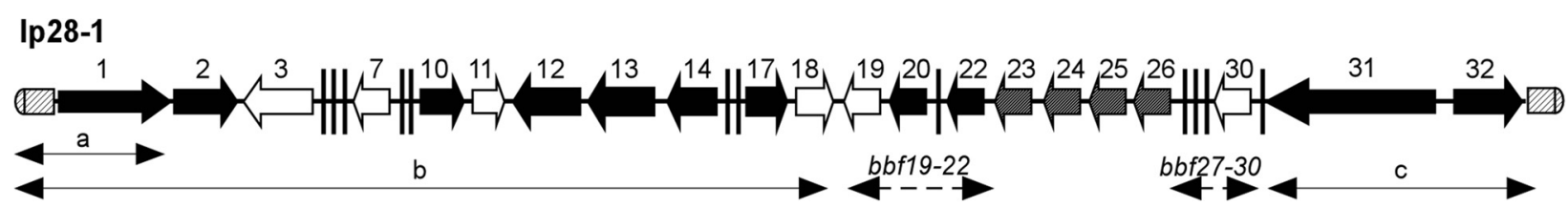

Fig. 1 Schematic of the open reading frames of the B. burgdorferi lp28-1 plasmid. Genes are flanked by telomeres (striped regions). Double-headed solid arrows show regions that have been previously deleted: a [11], b and c [8]. Genetic loci bbf19-22 or bbf27-30 were chosen for mutational analysis, as there is no knowledge for their respective roles in B. burgdorferi persistence. Conserved regions (black); pseudogenes (white); short genes less than $300 \mathrm{bp}$ (vertical lines); autonomous replication genes (grey)

infectivity and persistence. Mutation or deletion of the bbf01 gene that encodes for the arthritis related protein, Arp, was shown to exhibit a reduced ability to cause joint inflammation, but did not alter infectivity or persistence in immunocompetent mice $[10,11]$. Clones containing a deletion of $b b f 01$ to $b b f 18$ were found to be fully infectious and persistent following infection in immunocompetent mice [8]. However, signature-tagged transposon mutagenesis of lp28-1 [12] identified several genes within the $b b f 1-18$ region ( $b b f 03, b b f 05, b b f 10$, and bbf18) as having a possible impact on infectivity. Region $b b f 23-b b f 26$ of lp28-1 codes for the paralogous family proteins 49, 32, 50 and 57, respectively. These genes are known to be required for autonomous replication of the lp28-1 plasmid [13], and have been shown to be required for infectivity [12]. In addition to previous mutational studies, work by Embers et al. found that regulation of outer surface protein $\mathrm{C}(\mathrm{OspC})$ is impaired in spirochetes lacking lp28-1, which could also potentially contribute to the lack of persistence exhibited by this strain [14]. This disregulation resulted in continued expression of OspC, which has been shown to lead to spirochete clearance by an adaptive host immune response [15-18].

To date, with the exception of $b b f 29$ [19], two regions containing the ORFs bbf19 - bbf22 and bbf27 - bbf30 have not been mutated in their entirety. Hence, the question addressed in this study is whether loss of the genes within these two regions has an effect on infectivity and persistence by $B$. burgdorferi. The bbf19-22 region encodes one pseudogene (bbf19), a conserved hypothetical protein (bbf20), a short gene (bbf21) and a putative p23 protein (bbf23). Region bbf27-30 encodes mostly short genes (bbf27-29) and one pseudogene (bbf30) [20]. To further investigate the role of non- $v l s$ genes of 1 28-1 in disease persistence and potentially the regulation of $\operatorname{ss} \mathrm{C}$ expression, mutants were generated that lacked either region $b b f 1-18, b b f 19-22$ or $b b f 27-30$, and their respective impact on infectivity and disease persistence was assessed in immunocompetent $\mathrm{C} 3 \mathrm{H}$ mice. Results provided here show no significant difference in infectivity and persistence between wild type $B$. burgdorferi and the generated mutants. In addition, the particular lp28-1 regions under study may not be involved in OspC downregulation as a mechanism of immune avoidance, as there was no significant difference in $\operatorname{sp} C$ expression levels during host infection between the wild-type and mutant clones.

\section{Methods}

\section{B. burgdorferi clones and culture methods}

Borrelia burgdorferi B31-A3 (wild type) was a kind gift from Patti Rosa [21]. Clones described in this study were generated from the above-mentioned B31 clone (Table 1). All B. burgdorferi clones were cultivated in liquid BSK-II medium supplemented with $6 \%$ rabbit serum (Cedarlane Laboratories, Burlington, NC) and incubated at $35{ }^{\circ} \mathrm{C}$ under $2.5 \% \mathrm{CO}_{2}$. Mutant strains were grown in media supplemented with kanamycin $(200 \mu \mathrm{g} / \mathrm{ml})$. Cell densities and growth phase were monitored by dark-field microscopy and enumerated using a Petroff-Hausser counting chamber. Blood and tissue samples were cultured in BSKII supplemented with $6 \%$ rabbit serum and containing Borrelia antibiotic cocktail $(0.02 \mathrm{mg} / \mathrm{ml}$ phosphomycin, $0.05 \mathrm{mg} / \mathrm{ml}$ rifampicin and $2.5 \mu \mathrm{g} / \mathrm{ml}$ amphotericin B).

\section{Generation of Ip28-1 mutant clones}

The mutant lacking regions $b b f 1-18$ was regenerated through targeted deletion as described in a previous publication [8]. For deletion of bbf19-22 and bbf27-30 from lp28-1 by allelic exchange, a 500 bp sequence upstream and downstream of each region to be deleted was PCR amplified from the annotated lp28-1 sequence (NCBI Reference Sequence: NC_001851.2 http://www.ncbi.nlm.nih.gov/) using primers listed in Table 2. The resulting DNA product was then cloned

Table 1 B. burgdorferi clones used in this study

\begin{tabular}{|c|c|c|}
\hline B. burgdorferi B31 clone & Missing plasmids & Reference \\
\hline A3 wild type & cp9 & [21] \\
\hline A3 $\Delta b b f$ 1-18 (Bb $\Delta 1-18)$ & cp9 & {$[8]$} \\
\hline A3 $\Delta b b f$ 19-22 (Bb $\Delta 19-22)$ & cp9 & This study \\
\hline A3 $\Delta b b f$ 27-30 (Bb $19-22)$ & cp9 & This study \\
\hline $5 A 8(B b \Delta / p 28-1)$ & Ip28-1 & [6] \\
\hline
\end{tabular}


Table 2 Primers used in this study

\begin{tabular}{|c|c|c|}
\hline P330 & GTCTGTGGTAGTTACTAGTTACTITAAATACC & Forward primer for bbf27-30 target \\
\hline P331 & CCGAAATATTCCTATCTACTTAACAAC & Reverse primer for bbf27-30 target \\
\hline P332 & CCGGCCGGCGAATITTAGTCCTCTAGTGAGTTGTG & Left primer for inverse PCR of bbf27-30 target in pJET with NgoMIV site \\
\hline P333 & CCGGCTAGCGTTATAAGCCCTCCATTTGATAATTITITG & Right primer for inverse PCR of bbf27-30 target in pJET with Nhel site \\
\hline P366 & CTTAATTTGGACCGCCATTAGAGC & Forward primer for bbf27-30 screen \\
\hline P367 & GGGTTITTGAAACAAATCTTGC & Reverse primer for $b b f 27-30$ screen \\
\hline P411 & GAGTTTCTGGTAAGATTAATGCTC & Forward primer for flaB RT-qPCR \\
\hline P412 & CATTTAAATTCCCTTCTGTTGTCTGA & Reverse primer for flaB RT-qPCR \\
\hline P413 & AGAGGTTTGTCACAAGCTTCTAGAAATACTTCAAAGGC & flaB RT-qPCR probe \\
\hline P482 & GAACAAGCTGAAAAATATAAAAAAGTAATG & Forward primer for PCR amplification of bbf19-22 target \\
\hline P483 & CTGGTTACTIITTAGATAGAGTIITTATAGAG & Reverse primer for PCR amplification of bbf19-22 target \\
\hline P484 & CCGGCCGGCGGTTTAGACTTGCATTAA TATCTCC & Left primer for inverse PCR bbf19-22 target in pJET with NgoMIV site \\
\hline P485 & CCGGCTAGCCCCCTCCTTATATIIITITATATATAAAAG & Right primer for inverse PCR of bbf19-22 target in pJET with Nhel site \\
\hline P486 & GCTTATAAGCTITATTAACACCCATATATTC & Forward primer for bbf19-22 screen \\
\hline P487 & CCCGCGAGGTATATTTATTTATATTG & Reverse primer for bbf19-22 screen \\
\hline P709 & TTACGGATTCTAATGCGGTTT & Forward primer for ospC RT-qPCR \\
\hline P710 & TTTACCAATAGCTTTAGCAGCAA & Reverse primer for ospC RT-qPCR \\
\hline P711 & TGTGAAAGAGGTTGAAGCGTTGCTG. & ospC RT-qPCR probe \\
\hline P828 & CTGCACTACCACAAGAGATTGCA & Forward primer for PCR screen for left-end sequence of Ip28-1 \\
\hline P829 & СТСТтСТССТСТСТTСТTСТСТСТ & Reverse primer for PCR screen for left-end sequence of Ip28-1 \\
\hline P830 & CATTTCTAGTCTAGATTGCAGTTATTTCTAAAATTAACT & Forward primer for PCR amplification for Ip28-1 left-end deletion target \\
\hline P831 & GTGCCCAGGCGGCCGTCCTTATTCTTCTGGCATAGAAGT & Reverse primer for PCR amplification for Ip28-1 left-end deletion target \\
\hline
\end{tabular}

into the pJET 2.1 plasmid vector (Fermentas, USA). Primers annealing within the $500 \mathrm{bp}$ region were then used to amplify the DNA vector excluding the regions to be deleted. An insert containing the aphI gene conferring resistance to kanamycin driven by the $f l g B$ promoter of B. burgdorferi was then ligated to this PCR product to create the plasmid DNA construct for allelic exchange (pPH17 or pPH30 for the deletion of $b b f 27-30$ and bbf19-22, respectively). The resulting plasmid was then transformed into Escherichia coli DH5 $\alpha$ competent cells. Plasmid DNA isolated from individual E. coli clones was verified for correct size and orientation by restriction digest before transformation into B. burgdorferi cells.

\section{B. burgdorferi transformation}

B. burgdorferi B31-A3 wild-type cells were electroporated and cultured as previously described [8, 22]. DNA from culture-positive wells was extracted using a DNeasy Blood and Tissue Kit (Qiagen, Germantown, $\mathrm{MD})$, and used for PCR analysis to confirm the presence of the antibiotic-resistance gene and the absence of $b b f 1-18, \quad b b f 19-22$ or $b b f 27-30$ utilizing primer sets described in Table 2. Plasmid content for each verified transformant was determined by PCR using plasmid-specific primers as previously described [6].

\section{Southern Blot analysis}

Total plasmid DNA was extracted from B. burgdorferi clones using the Plasmid Midi Kit (Qiagen) and separated on a $1 \%$ agarose gel at $80 \mathrm{~V}$ for $23 \mathrm{~h}$ (250 $\mathrm{ng}$ of DNA was used per lane). DNA was then transferred onto a nylon membrane and hybridized with Digoxigenin (DIG)-labeled probes following manufacturer's guidelines (Roche, Indianapolis, IN).

\section{Infection of mice}

Three to four week-old male $\mathrm{C} 3 \mathrm{H} / \mathrm{HeN}$ mice (Harlan, Indianapolis, IN) were infected by subcutaneous needle inoculation with $10^{5}$ total spirochetes per dose. B. burgdorferi clones from frozen glycerol stock were passaged no more than two times in vitro prior to use in mouse infection assays. Infection was monitored by culturing either blood samples or ear biopsies at the indicated times post infection. After infection, mice were monitored for presence of infection at day seven by blood collection. Tissues for RT-qPCR analysis were collected at days 14, 21, 56 and 91. Two ear punch biopsies of about $2 \mathrm{~mm}$ diameter were collected from each mouse and 
stored in RNA later in $-20^{\circ} \mathrm{C}$ until RNA extraction was done.

\section{RNA extraction and gene expression analysis by droplet digital PCR}

RNA was extracted from the tissues using the RNeasy Fibrous Tissue Mini Kit (Qiagen, Valencia, CA) and treated with the RNase-free DNase set (Qiagen) to remove any residual DNA. The concentration, purity and integrity of RNA were determined electrophoretically and by measuring the absorbance. The reverse transcription reaction was performed in a total volume of $20 \mu \mathrm{L}$ using iScript Advanced cDNA Synthesis Kit for RTqPCR (Biorad) following manufacturers guidelines. A quantity of $100 \mathrm{ng}$ total RNA was used as template. Following generation of CDNA, droplet digital PCR was performed using the QX100 Droplet Digital PCR $\left(\mathrm{ddPCR}^{\mathrm{mm}}\right)$ system (Bio-Rad). The ospC and flaB genes were amplified using primers and probes shown in Table 2. The primer probe mixture, cDNA and $1 \mathrm{x}$ ddPCR supermix were pipetted into the DG8 ${ }^{\mathrm{rm}}$ Cartridge for droplet formation in the QX100 droplet generator. Droplets were then transferred into a 96 well PCR plate and heat-sealed with a foil plate seal (Biorad) before placement into the C1000 Touch Thermal Cycler. Reactions were performed in biological replicates in a total volume of $40 \mu \mathrm{l}$. Cycling parameters (with ramping speed at $2{ }^{\circ} \mathrm{C} \mathrm{s}^{-1}$ ) were as follows: enzyme activation was done at $95{ }^{\circ} \mathrm{C}$ for $10 \mathrm{~min}$ followed by a denaturation for $30 \mathrm{~s}$ at $94{ }^{\circ} \mathrm{C}$; annealing and extension for $1 \mathrm{~min}$ at $60{ }^{\circ} \mathrm{C}$ was repeated for 40 cycles. After PCR, the plate was placed in the QX100 droplet reader and relative gene expression determined using the QuantaSoft software.

\section{Statistical analysis}

SigmaPlot 11.0 software was used for all statistical data analysis. Normalized ospC transcript values from mice infected with the mutant clones were compared to transcript levels from mice infected with the wild type clone on respective days tested. Results were analyzed by the Student's $t$-test and $p$ values $\leq 0.05$ were considered statistically significant.

\section{Results}

Generation of Ip28-1 mutant B. burgdorferi clones

Genetic loci of the lp28-1 plasmid containing the genes bbf19-22 and bbf27-30 (see Fig. 1) were chosen for mutational analysis to assess their respective roles in $B$. burgdorferi persistence. Deletion of each genetic region was achieved using allelic exchange as shown in Fig. 2a. Deletion constructs (pPH17 and pPH30) were generated that carried a kanamycin-resistance gene (kan) driven by
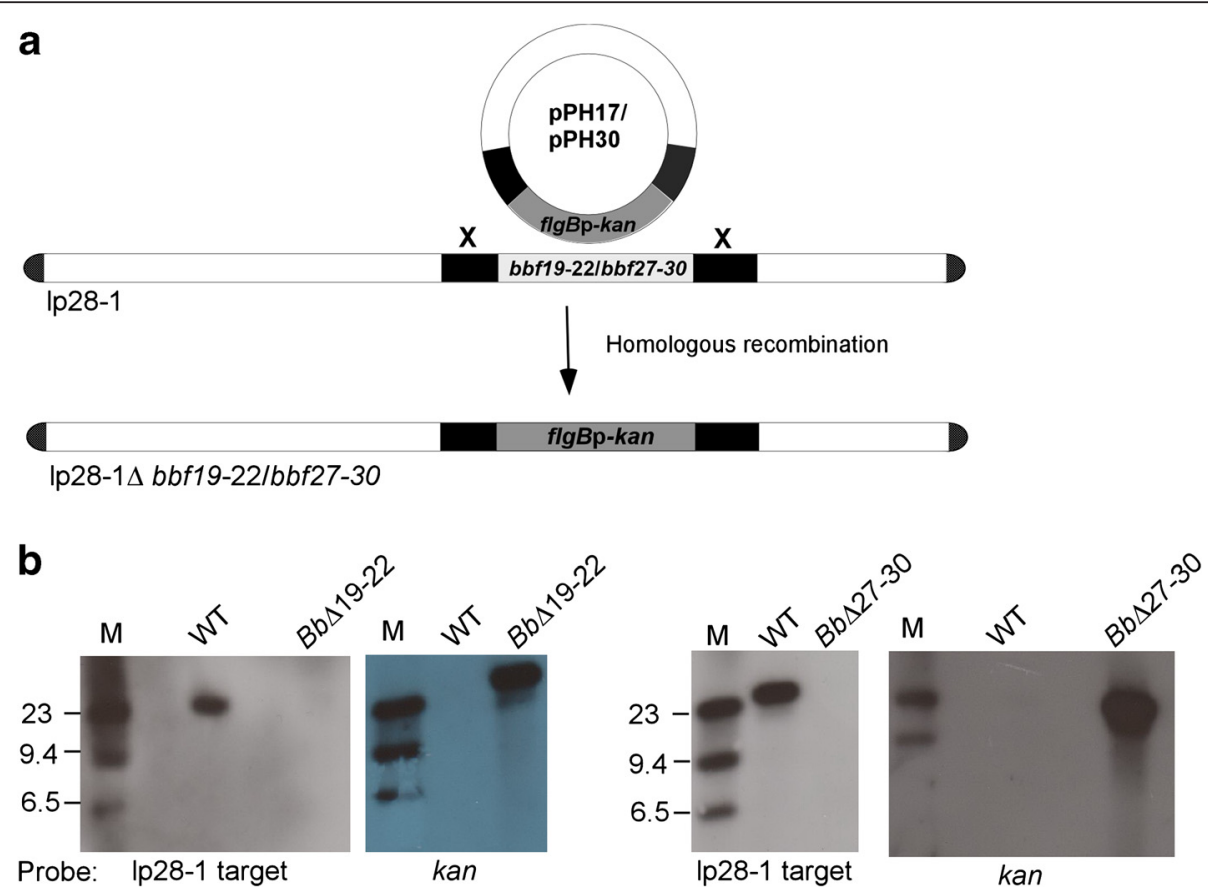

Fig. 2 Generation and verification of Ip28-1 mutant clones of B. burgdorferi. a Schematic of the construction strategy for the Ip28-1 mutant clones

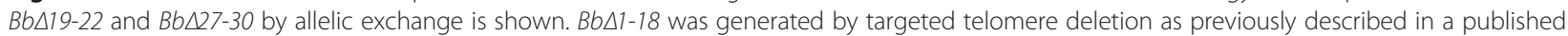
study [8]. To confirm the loss of regions bbf19-22 or bbf27-30, DNA was isolated and subjected to Southern blot analysis (b). All mutant clones provided a positive signal of the expected size $(\sim 28 \mathrm{~kb})$ when probed for the kan cassette. The blot also confirmed the absence of the targeted region in the two mutant clones. WT, wild type; M, DNA size marker 
a B. burgdorferi flgB promoter flanked by $500 \mathrm{bp}$ homologous sequences upstream and downstream of the targeted region. Genetic deletion of the $b b f 19-22$ and bbf27-30 regions was achieved by insertion of the selectable kan marker by homologous recombination to generate the mutant clones, $B b \Delta 19-22$ and $B b \Delta 27-30$, respectively. Transformants were PCR screened for the presence of the kanamycin-resistance cassette, and clones meeting this criterion were then further analyzed for their total plasmid profile. All clones contained the full plasmid profile, with the exception of cp9. The cp9 plasmid is normally absent from the parental B31-A3 clone, and is not necessary for infection or pathogenesis [21]. To confirm the loss of each region, DNA was isolated from $B b \Delta 19-22$ and $B b \Delta 27-30$ and subjected to Southern blot analysis (Fig. 2b). A B. burgdorferi mutant lacking $b b f 1-18$ was also generated and verified as previously reported [8]. This mutant was included because previous infection studies were only carried out for 28 days, and the intention of this study was to prolong infection for 91 days to assess for long-term infection.

\section{Lp28-1 mutant clones are capable of dissemination and long-term infection in immunocompetent mice}

To assess the individual infectivity profiles of the newly generated mutants, groups of six $\mathrm{C} 3 \mathrm{H} / \mathrm{HeN}(\mathrm{C} 3 \mathrm{H})$ mice each were needle inoculated with either $B b \Delta 1-18$, $B b \Delta 19-22$ or $B b \Delta 27-30$ clones. Two groups of three $\mathrm{C} 3 \mathrm{H}$ mice each were infected with wild type or lp28-1deficient $(B b \Delta l p 28-1)$ B. burgdorferi clones to serve as positive and negative controls, respectively. As shown in Table 3, blood samples collected at day 7 post infection produced positive cultures for spirochetes in all inoculated mouse groups except with the BbD19-22 mutant. Positive blood cultures were obtained from only 3 out of 6 mice infected with the $B b \Delta 19-22$ clone. This pattern was also observed for this same group of mice at day 14 after culture of ear biopsies, suggesting a possible delay in dissemination to this tissue site. At day 14 post infection, cultures were positive for spirochetes in mice

Table 3 Infectivity of B. burgdorferi clones in $\mathrm{C} 3 \mathrm{H}$ mice

\begin{tabular}{|c|c|c|c|c|c|c|c|c|}
\hline \multicolumn{9}{|l|}{ Days post infection: } \\
\hline \multirow[b]{2}{*}{ B. burgdorferi clone } & \multirow{2}{*}{$\begin{array}{l}7 \\
\text { Blood }\end{array}$} & \multirow{2}{*}{$\begin{array}{l}14 \\
\text { Ear }\end{array}$} & \multirow[t]{2}{*}{21} & \multirow[t]{2}{*}{56} & \multicolumn{4}{|l|}{91} \\
\hline & & & & & Heart & Bladder & Ear & Joint \\
\hline Wild type & $3 / 3^{*}$ & $3 / 3$ & $3 / 3$ & $3 / 3$ & $3 / 3$ & $3 / 3$ & $3 / 3$ & $3 / 3$ \\
\hline$B b \Delta 7-18$ & $6 / 6$ & $6 / 6$ & $6 / 6$ & $6 / 6$ & $6 / 6$ & $6 / 6$ & $6 / 6$ & $6 / 6$ \\
\hline$B b \triangle 19-22$ & $3 / 6$ & $3 / 6$ & $6 / 6$ & $6 / 6$ & $6 / 6$ & $6 / 6$ & $6 / 6$ & $6 / 6$ \\
\hline$B b \triangle 27-30$ & $6 / 6$ & $5 / 6$ & $6 / 6$ & $6 / 6$ & $6 / 6$ & $6 / 6$ & $6 / 6$ & $6 / 6$ \\
\hline$B b \Delta / p 28-1$ & $3 / 3$ & $1 / 3$ & $0 / 3$ & $0 / 3$ & $0 / 3$ & $0 / 3$ & $0 / 3$ & $0 / 3$ \\
\hline
\end{tabular}

Values listed correspond to numbers of positive cultures/number of mice tested. Six or three mice were used for each $B$. burgdorferi clone infected with wild type, $B b \Delta 1-18$ and $B b \Delta 27-30$. As expected, spirochetes could not be detected from tissues collected from day 14 onwards from mice infected with the $B b \Delta l p 28-1$ clone due to the lack of lp28-1 [8]. Longterm infection ( $>21$ days) was conducted to assess whether the $B b \Delta 1-18, B b \Delta 19-22$ and $B b \Delta 27-30$ mutant clones would eventually be cleared in infected mice. Ear biopsies were collected at days 21 and 56 post infection, and mice were sacrificed at day 91 to harvest heart, bladder, ear, and joint tissues for spirochete culture. Ear biopsies and cultured tissues from all mice were found to be culture positive, demonstrating that the $B b \Delta 1-18$, $B b \Delta 19-22$ and $B b \Delta 27-30$ clones were fully capable of dissemination and long-term persistent infection (Table 3).

\section{Lp28-1 mutants are capable of downregulating ospC expression during host infection}

Associated with the development of the host-acquired immune response is OspC downregulation and increased synthesis of VlsE on lp28-1 [23, 24]. To determine if the deletion of $b b f 1-18, b b f 19-22$ or $b b f 27-30$ has an effect on $\operatorname{ssp} C$ expression during infection of a murine host, groups of $3 \mathrm{C} 3 \mathrm{H}$ mice each were needle inoculated with $10^{5}$ spirochetes of either $B b \Delta 1-18, B b \Delta 19-22$ or $B b \Delta 27$ 30. Two additional groups were infected with wild type or $B b \Delta l p 28-1$ clones to serve as controls. For each group, mice were infected for a duration of either 21 or 91 days; these time points were chosen to assess ospC expression levels during early and late stages of infection, respectively. On day 21 and 91 post infection, heart and bladder tissue samples were collected for RNA extraction. Ear biopsy tissue was also collected on days 13, 21, 56 and 91 for RNA extraction as a way to assess the osp $C$ transcription profile during infection without sacrificing mice. Expression of $o s p C$ was normalized against the $f l a B$ housekeeping gene and compared to mRNA of late log phase in vitro-grown B. burgdorferi wild type.

Relative expression levels of ospC transcripts were determined by digital droplet PCR (Fig. 3). FlaB and ospC transcripts were detected at day 13 in tissues collected from mice infected with $B b \Delta l p 28-1$ clone, which correlated with culture results (see Table 3). Data for this clone was not collected after day 21 as all mice successfully cleared infection after this time point. Relative levels of $o s p C$ expression between tissues infected with the wild type and mutant clones were compared using a Student's $t$-test. Results presented in Fig. 3a show normalized $o s p C$ expression from spirochetes in bladder tissue relative to $f l a B$ transcripts. No significant difference was observed between the wild type and mutant clones recovered from bladder tissues at day 21 . At day 91 post infection, $o s p C$ expression by $B b \Delta 1-18$ spirochetes collected from bladder tissues of mice was significantly 

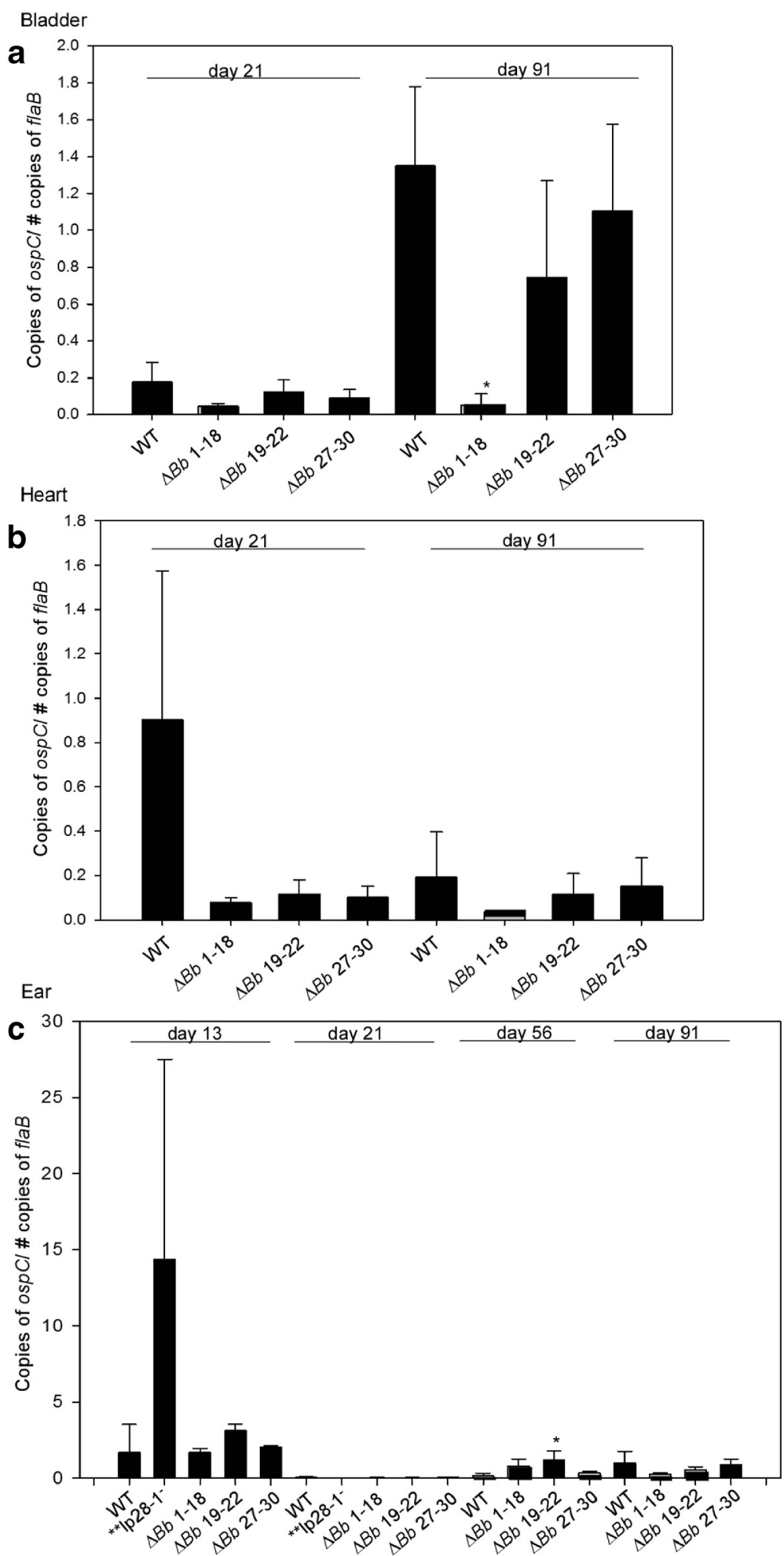

Fig. 3 (See legend on next page.) 
(See figure on previous page.)

Fig. 3 qRT-PCR analysis of ospC transcript levels in various tissue sites of infected mice. ospC expression profile of spirochetes recovered from the

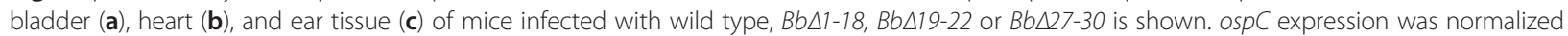
against flaB and compared to mRNA of in vitro-grown B. burgdorferi wild-type cells grown to late log phase. Asterisks show mutant clones with ospC expression levels that were statistically different from the wild type using statistical analyses described in Methods. a) In bladder tissue, no difference in ospC expression was observed at day 21. At day 91, spirochetes from wild type-infected tissues had a statistically significant difference when compared to $B b \Delta 1-18$ infected tissues $(p=0.007)$. b) Spirochetes in heart tissue samples showed no significant difference when compared to wild type-infected tissues at both days 21 and 91. c) Spirochetes in the ear tissue showed a general decline in ospC expression from day 13, which was lowest at day 21 and started to rise at day 56. There was no significant difference in ospC expression noted at day 13, 21 and 91 . At day 56, spirochetes from mice infected with $B b \Delta 19-22$ had a higher expression of osp $C$ when compared to those infected by the wild type $(p=0.043)$. ${ }^{*}$ flaB and ospC transcripts were detected at day 13 in tissues collected from mice infected with $B b \Delta / p 28-1$ clone, which correlated with culture results (see Table 3). Data for this clone was not collected after day 21 as all mice successfully cleared infection after this time point

repressed relative to that from wild type-infected bladders $(p=0.007)$. No statistical difference was noted with the other clones. Spirochetes recovered from heart tissue at both day 21 and 91 showed no significant difference in $\operatorname{sspC}$ expression (Fig. 3b).

For ear tissue, normalized $\operatorname{ss} \mathrm{C}$ expression could be determined from day 13 onwards (Fig. 3c). No significant difference in ospC expression was observed between wild type and the mutant clones recovered from infected ear tissues collected at day 13, 21 and 91 . At day 56 post infection, the $B b \Delta 19-22$ clone was found to have a significantly higher level of ospC expression compared to the wild type $(p=0.043)$, while no difference was noted with the other two mutant clones. Taken together, the results from the mice infection studies and RT-qPCR analysis suggest that the lp28-1 regions targeted for deletion are not important for persistent infection by B. burgdorferi, and have little to no lasting effects on the expression/repression of $o s p C$.

\section{Discussion}

Gene conversion at the vls locus on lp28-1 results in antigenic variation of VlsE, which has been demonstrated repeatedly to be essential for immune evasion and persistence by the Lyme disease pathogen $[2,3,8,9$, $25]$. The question remained whether lack of the $v l s$ locus alone explains the intermediate infectivity phenotype exhibited by clones lacking the lp28-1 plasmid. Previous studies have excluded all remaining genes as having an effect on persistent infection, with the exception of the genetic loci bbf19-22 and bbf27-30 [8, 9]. A B. burgdorferi mutant lacking $b b f 1-18$ that was previously reported [8] was included in the present study because previous infection studies were only carried out for 28 days, and the intention of this study was to prolong infection for 91 days to assess long-term persistence. The $b b f 1-18$ region has several conserved coding regions, short genes, and pseudogenes. In addition, a number of genes in this region (bbf03, bbf05, bbf10, and bbf18) have been identified as potentially important for infectivity [12]. The bbf19-22 region encodes one pseudogene (bbf19), a conserved hypothetical protein (bbf20), a short gene (bbf21) and a putative p 23 protein (bbf23). Region $b b f 27-30$ encodes mostly short genes (bbf27-29) and one pseudogene (bbf30; [20]. Although the possibility has been raised that one or more of these genes could be involved in infectivity or persistence, the data reported here demonstrate that lp28-1 mutant clones lacking bbf1-18, bbf1922 or $b b f 27-30$ were fully capable of tissue dissemination and long-term infection of an immunocompetent murine host. This strongly suggests that these non- $v l s$ genes of lp28-1 do not play a role in persistent infection by the Lyme disease pathogen.

A previous study suggested that failure of immune evasion by clones lacking lp28-1 might also be due to altered expression of the surface lipoprotein, OspC. It was reported that $\operatorname{osp} C$ expression by $\mathrm{lp} 28-1^{-}$spirochetes was abnormally high, suggesting an impairment in downregulation of this protein [14]. OspC is required for the establishment of mammalian infection and is an effective immune target that has to be downregulated to avoid immune clearance [18, 26, 27]. Regulation of antigen expression has been shown to be essential for spirochete survival within the complex enzootic cycle involving the tick and mammal [16, 23, 28-30]. OspC production increases when the tick feeds [30,31], and has been shown to be required for $B$. burgdorferi to establish infection in the mammal [26, 27, 32-34]. However, OspC is dispensable during persistent infection [27, 35 , and it has been reported that its continued expression in an immunocompetent animal is detrimental to $B$. burgdorferi survival [18]. This suggests that B. burgdorferi must downregulate OspC following a specific humoral response as a strategy to evade [28] host immunity and ensure persistence in the mammalian host. Failure of $\mathrm{OspC}$ downregulation by spirochetes lacking lp28-1 has been previously shown, and it has been suggested that OspC regulation by genes on this plasmid may be a potential mechanism of immune evasion [14].

Despite the observed ability of the mutant clones in the present study to persistently infect mice, an additional component of this study was to assess whether genes within the $b b f 01-22$ or $b b f 27-30$ regions are potentially responsible for the increased ospC expression 
previously observed for spirochetes lacking lp28-1. FlaB and $o s p C$ mRNA transcripts were consistently detected from ear tissues at day 13 post infection. At day 12, it has been shown that $o s p C$ is abundantly expressed in the skin compared to the heart and joint [17], making the skin a reliable tissue to observe $o s p C$ expression during early infection without sacrificing the animal. AntiOspC antibody is detectable at day 10 , and then peaks at days 24-45 before starting to decline [15]. Consistent with this timeline, the results showed relatively high levels of $o s p C$ expression in the ear at day 13 post infection when humoral immunity against $\mathrm{OspC}$ is not fully established (Fig. 3c). By day 21 post infection, ospC transcript levels from all tissue samples collected from mice infected with mutant or wild type spirochetes were greatly reduced suggesting that the mutations generated within lp28-1 did not affect OspC down regulation. This is indicative of either immune selection against spirochetes that abundantly express this lipoprotein and/or persistence of spirochetes that decrease its expression. Moreover, a general increase in $o s p C$ expression by both the wild type and mutant clones was observed in infected ear and bladder tissues at days 56 and 91, which coincides with the reported decline of anti-OspC antibody [15] (Fig. 3a and c).

Overall, relatively comparable ospC expression levels were observed for the wild type and mutant clones. The wild type clone generally showed higher levels of ospC expression, with the exception of day 56 when $B b \Delta 19-22$ mutant spirochetes colonizing ear tissue exhibited significantly higher expression levels. Initial delay in tissue colonization was also observed with this mutant at days 7 and 14 post infection. In the end, the higher ospC expression levels did not correspond to clearance of spirochete infection as demonstrated by the long-term mouse infection studies.

From the data presented in this study, the lp28-1 regions targeted for deletion are not important for persistent infection by $B$. burgdorferi, and have little to no lasting effects on the expression/repression of ospC. However, it is conceivable that the regions under study may have a detectable phenotype in mice when given a lower inoculum dose. Moreover, it is possible that these regions could play a role in other aspects of infectivity; both possibilities will require further study.

\section{Conclusions}

The data reported herein indicate that regions $b b f 1-18$, bbf19-22 and bbf27-30 of lp28-1 are not involved in persistent infection by $B$. burgdorferi, and do not critically alter the overall expression levels of osp $C$ during murine infection leading to immune clearance. Moreover, along with the previous data involving mutation of non-vls loci on lp28-1, these findings suggest that the $v l s$ locus may be the sole genetic element on this plasmid responsible for immune evasion and persistence exhibited by the Lyme disease pathogen.

\section{Acknowledgments \\ The authors thank the Call laboratory in the Paul G. Allen School for Global Animal Health at Washington State University for the use of the digital droplet instrument, and are indebted to Lisa Orfe for providing excellent technical assistance. We also thank Allison James, Abdul Lone, and Jessica Wong for critical reading of the manuscript.}

\section{Funding}

This work was funded by an intramural grant (17A-2540-9830) by the College of Veterinary Medicine at Washington State University.

\section{Availability of data and material}

The datasets generated during and/or analyzed during the current study are available from the corresponding author on reasonable request.

\section{Authors' contributions}

PHM carried out all experiments, contributed to data organization, analysis and interpretation of the results, and writing of the final manuscript. TB contributed to the design of the study, interpretation of the results, and assisted in the drafting of the manuscript. All authors have read and approved the final manuscript.

\section{Competing interests}

The authors declare that they have no competing interests.

\section{Consent for publication}

Not applicable.

\section{Ethics approval and consent to participate}

The experimental procedures involving Mus musculus strains of inbred mice were carried out in accordance with the American Association for Accreditation of Laboratory Animal Care (AAALAC) protocol and the institutional guidelines set by the Office of Campus Veterinarian at Washington State University (Animal Welfare Assurance A3485-01 and USDA registration number 91-R-002). Washington State University AAALAC and institutional guidelines are in compliance with the U.S. Public Health Service Policy on Humane Care and Use of Laboratory Animals. Mus musculus inbred mice were maintained at Washington State University (Pullman, WA, USA) in an AAALAC-accredited animal facility. The Washington State University Institutional Animal Care and Use Committee reviewed and approved the animal protocols associated with the current studies.

Received: 7 May 2016 Accepted: 4 August 2016

Published online: 08 August 2016

\section{References}

1. Bhate C, Schwartz RA. Lyme disease: Part I. Advances and perspectives. J Am Acad Dermatol. 2011;64(4):619-36.

2. Zhang JR, Hardham JM, Barbour AG, Norris SJ. Antigenic variation in Lyme disease borreliae by promiscuous recombination of VMP-like sequence cassettes. Cell. 1997:89(2):275-85.

3. Zhang JR, Norris SJ. Genetic variation of the Borrelia burgdorferi gene vIsE involves cassette-specific, segmental gene conversion. Infect Immun. 1998;66(8):3698-704.

4. Labandeira-Rey M, Seshu J, Skare JT. The absence of linear plasmid 25 or 28-1 of Borrelia burgdorferi dramatically alters the kinetics of experimental infection via distinct mechanisms. Infect Immun. 2003;71(8):4608-13.

5. Labandeira-Rey M, Skare JT. Decreased infectivity in Borrelia burgdorferi strain B31 is associated with loss of linear plasmid 25 or 28-1. Infect Immun. 2001;69(1):446-55.

6. Purser JE, Norris SJ. Correlation between plasmid content and infectivity in Borrelia burgdorferi. Proc Natl Acad Sci U S A. 2000;97(25):13865-70.

7. Purser JE, Lawrenz MB, Caimano MJ, Howell JK, Radolf JD, Norris SJ. A plasmid-encoded nicotinamidase (PnCA) is essential for infectivity of Borrelia burgdorferi in a mammalian host. Mol Microbiol. 2003;48(3):753-64. 
8. Bankhead T, Chaconas $\mathrm{G}$. The role of VlsE antigenic variation in the Lyme disease spirochete: persistence through a mechanism that differs from other pathogens. Mol Microbiol. 2007;65(6):1547-58.

9. Rogovskyy AS, Bankhead T. Variable VlsE is critical for host reinfection by the Lyme disease spirochete. PLoS One. 2013;8(4):e61226.

10. Imai D, Holden K, Velazquez EM, Feng S, Hodzic E, Barthold SW. Influence of arthritis-related protein (BBF01) on infectivity of Borrelia burgdorferi B31. BMC Microbiol. 2013;13(1):100.

11. Hove PR, Haldorson GJ, Magunda F, Bankhead T. Presence of arp specifically contributes to joint tissue edema associated with early-onset lyme arthritis. Infect Immun. 2014;82(1):43-51.

12. Lin T, Gao L, Zhang C, Odeh E, Jacobs MB, Coutte L, Chaconas G, Philipp MT, Norris SJ. Analysis of an ordered, comprehensive STM mutant library in infectious Borrelia burgdorferi: insights into the genes required for mouse infectivity. PLoS One. 2012;7(10):e47532.

13. Stewart PE, Chaconas G, Rosa P. Conservation of plasmid maintenance functions between linear and circular plasmids in Borrelia burgdorferi. J Bacteriol. 2003;185(10):3202-9.

14. Embers ME, Alvarez X, Ooms T, Philipp MT. The failure of immune response evasion by linear plasmid 28-1-deficient Borrelia burgdorferi is attributable to persistent expression of an outer surface protein. Infect Immun. 2008;76(9):3984-91.

15. Liang FT, Jacobs MB, Bowers LC, Philipp MT. An immune evasion mechanism for spirochetal persistence in Lyme borreliosis. J Exp Med. 2002;195(4):415-22.

16. Liang FT, Nelson FK, Fikrig E. Molecular adaptation of Borrelia burgdorferi in the murine host. J Exp Med. 2002;196(2):275-80.

17. Liang FT, Yan J, Mbow ML, Sviat SL, Gilmore RD, Mamula M, Fikrig E. Borrelia burgdorferi changes its surface antigenic expression in response to host immune responses. Infect Immun. 2004;72(10):5759-67.

18. Xu Q, Seemanapalli SV, McShan K, Liang FT. Constitutive expression of outer surface protein C diminishes the ability of Borrelia burgdorferi to evade specific humoral immunity. Infect Immun. 2006;74(9):5177-84

19. Grimm D, Eggers CH, Caimano MJ, Tilly K, Stewart PE, Elias AF, Radolf JD, Rosa PA. Experimental assessment of the roles of linear plasmids Ip25 and Ip28-1 of Borrelia burgdorferi throughout the infectious cycle. Infect Immun. 2004;72(10):5938-46.

20. Fraser CM, Casjens S, Huang WM, Sutton GG, Clayton $R$, Lathigra $R$, White $O$, Ketchum KA, Dodson R, Hickey EK, et al. Genomic sequence of a Lyme disease spirochaete, Borrelia burgdorferi. Nature. 1997:390(6660):580-6.

21. Elias AF, Stewart PE, Grimm D, Caimano MJ, Eggers CH, Tilly K, Bono JL, Akins DR, Radolf JD, Schwan TG, et al. Clonal polymorphism of Borrelia burgdorferi strain B31 Ml: implications for mutagenesis in an infectious strain background. Infect Immun. 2002;70(4):2139-50.

22. Samuels DS. Electrotransformation of the spirochete Borrelia burgdorferi. Methods Mol Biol. 1995;47:253-9.

23. Crother TR, Champion Cl, Whitelegge JP, Aguilera R, Wu XY, Blanco DR, Miller JN, Lovett MA. Temporal analysis of the antigenic composition of Borrelia burgdorferi during infection in rabbit skin. Infect Immun. 2004;72(9):5063-72.

24. Hodzic E, Feng S, Freet KJ, Barthold SW. Borrelia burgdorferi population dynamics and prototype gene expression during infection of immunocompetent and immunodeficient mice. Infect Immun. 2003;71(9):5042-55.

25. Zhang JR, Norris SJ. Kinetics and in vivo induction of genetic variation of vIsE in Borrelia burgdorferi. Infect Immun. 1998;66(8):3689-97.

26. Tilly K, Krum JG, Bestor A, Jewett MW, Grimm D, Bueschel D, Byram R, Dorward D, Vanraden MJ, Stewart P, et al. Borrelia burgdorferi OspC protein required exclusively in a crucial early stage of mammalian infection. Infect Immun. 2006;74(6):3554-64.

27. Seemanapalli SV, Xu Q, McShan K, Liang FT. Outer surface protein C is a dissemination-facilitating factor of Borrelia burgdorferi during mammalian infection. PLoS One. 2010;5(12):e15830.

28. Radolf JD, Samuels DS. Borrelia : molecular biology, host interaction, and pathogenesis. Norfolk, UK: Caister Academic Press; 2010.

29. Ohnishi J, Piesman J, de Silva AM. Antigenic and genetic heterogeneity of Borrelia burgdorferi populations transmitted by ticks. Proc Natl Acad Sci U S A. 2001;98(2):670-5.

30. Schwan TG, Piesman J, Golde WT, Dolan MC, Rosa PA. Induction of an outer surface protein on Borrelia burgdorferi during tick feeding. Proc Natl Acad Sci U S A. 1995;92(7):2909-13.
31. Schwan TG, Piesman J. Temporal changes in outer surface proteins $A$ and $C$ of the lyme disease-associated spirochete, Borrelia burgdorferi, during the chain of infection in ticks and mice. J Clin Microbiol. 2000;38(1):382-8.

32. Grimm D, Tilly K, Byram R, Stewart PE, Krum JG, Bueschel DM, Schwan TG, Policastro PF, Elias AF, Rosa PA. Outer-surface protein C of the Lyme disease spirochete: a protein induced in ticks for infection of mammals. Proc Natl Acad Sci U S A. 2004;101(9):3142-7.

33. Stewart PE, Wang $X$, Bueschel DM, Clifton DR, Grimm D, Tilly K, Carroll JA, Weis JJ, Rosa PA. Delineating the requirement for the Borrelia burgdorferi virulence factor OspC in the mammalian host. Infect Immun. 2006;74(6):3547-53.

34. Tilly K, Bestor A, Jewett MW, Rosa P. Rapid clearance of Lyme disease spirochetes lacking OspC from skin. Infect Immun. 2007;75(3):1517-9.

35. Tilly K, Bestor A, Rosa PA. Lipoprotein succession in Borrelia burgdorferi: similar but distinct roles for OspC and VIsE at different stages of mammalian infection. Mol Microbiol. 2013;89(2):216-27.

\section{Submit your next manuscript to BioMed Central and we will help you at every step:}

- We accept pre-submission inquiries

- Our selector tool helps you to find the most relevant journal

- We provide round the clock customer support

- Convenient online submission

- Thorough peer review

- Inclusion in PubMed and all major indexing services

- Maximum visibility for your research

Submit your manuscript at www.biomedcentral.com/submit
) Biomed Central 